\title{
Ultrafast quantum spin-state switching in the Co-octaethylporphyrin molecular magnet with a terahertz pulsed magnetic field
}

\author{
Oleg V.Farberovich ${ }^{1,2,3}$,Victoria L.Mazalova ${ }^{2}$, Valeri S.Stepanyuk ${ }^{4}$ \\ ${ }^{1}$ School of Physics and Astronomy, \\ Beverly and Raymond Sackler Faculty of Exact Sciences, \\ Tel Aviv University, Tel Aviv 69978, Israel \\ ${ }^{2}$ International Center "Smart Materials", \\ Southern Federal University, Zorge 5, \\ 344090, Rostov-on-Don, Russia \\ ${ }^{3}$ Voronezh State University, Voronezh 394000, Russia \\ 4 Max Planck Institute of Microstructure Physics, Halle, Germany
}

(Dated: June 25, 2015)

\begin{abstract}
Molecular spin crossover switches are the objects of intense theoretical and experimental studies in recent years. This interest is due to the fact that these systems allow one to control their spin state by applying an external photo-, thermo-, piezo-, or magnetic stimuli. The greatest amount of research is currently devoted to the study the effect of the photoexcitation on the bi-stable states of spin crossover single molecular magnets (SMMs). The main limitation of photo-induced bi-stable states is their short lifetime. In this paper we present the results of a study of the spin dynamics of the CoOEP molecule in the Low Spin (LS) state and the High Spin (HS) state induced by applying the magnetic pulse of $36.8 T$. We show that the spin switching in case of the HS state of the CoOEP molecule is characterized by a long lifetime and is dependent on the magnitude and duration of the applied field. Thus, after applying an external stimuli the system in the LS state after the spin switching reverts to its ground state, whereas the system in the HS state remains in the excited state for a long time. We found that the temperature dependency of magnetic susceptibility shows an abrupt thermal spin transition between two spin states at $40 \mathrm{~K}$. The proposed here theoretical approach opens the way to create modern devices for spintronics with the controllable spin switching process.
\end{abstract}

PACS numbers:

\section{INTRODUCTION}

Spin engineering mostly concentrates on the manipulation of spin degrees of freedom in various magnetic materials and the exploration of their potential applications in spintronics 1 . Current examples for the applications of spin engineering in magnetic nanostructures and devices are increasingly ample, ranging from the development of single molecular magnet as qubits ${ }^{2}$. Therefore, the spin engineering in magnetic nanostructures, including theoretical modeling, experimental demonstration, and device design for applications, is demanding multidisciplinary backgrounds of knowledge and technology which create great challenges for researchers.

Magnetic or spin logic appears as an appealing alternative due to its nonvolatile character, which can boost up switching on/off, its possibility to reduce the size of the element down to the several-atoms scale on/off spin per atom instead of one elementary charge per $10^{4}$ atoms in semiconductors, and speed increase as a secondary size effect ${ }^{3}$. The modern experiments are promising, they still move in the micrometer regime ${ }^{4}$, thus not fully exploiting the possible quantum nature of molecular magnetism. In other hand, small molecules perform logic operations using as input cation concentrations. The latter is fast with respect to the logic operation but slow with respect to repeatability. Thus a need for magnetic-logic devices on the molecular scale emerges. At the same time the experimental evidence of laser-driven ultrafast magnetic(spin) manipulation in the antiferromagnetic materials motivates the design of a cluster with more than one spin center which allows for spin manipulation ${ }^{5,6}$.

The problem of the gigahertz magnetization(spin) switching speed of todays magnetic logic and a magnetic memory devices into the terahertz regime underlies the entire field of information processing 7.8. The physics of present-day devices imposes serious limitations on this technological transformation, so we must invent new paradigms based on the quantum spin dynamics in the picosecond regime. This challenge could be met by a simulation of the quantum spin switching in a picosecond pulsed magnetic field ${ }^{9,10}$. Currently, no easily accessible method is available to generate intense sub-picosecond magnetic pulses localized at the nanoscale. However in the work ${ }^{11}$, show that bimetallic nanorings can act as nanoscale sources of intense ultrashort magnetic pulses. In this instance rely on the enhanced light absorption associated with the plasmons of metallic rings to generate transient thermoelectric currents that in turn produce sub-picosecond pulses with magnetic fields as high as a few tenths of a Tesla in the vicinity of the rings. The ability to generate strong magnetic fields localized on the nanoscale is of interest for elucidating spin and magnetization dynamics at sub-picosecond time and nanometer 
length scales $\frac{12}{2}$, and it holds great potential for materials characterization, terahertz radiation generation, and magnetic recording.

Magnetic molecules have been intensively studied so far because of their potential technological applications and for the possibility they offer to investigate fundamental properties of matter at the nanoscopic scale $\underline{13}$. An intensively studied class of magnetic molecules is constituted by the so called single molecule magnets or molecular nanomagnets. These systems are characterized by a slow relaxation of magnetization at low $T$ and give rise to magnetic hysteresis. This is one condition for storing information in a molecule. Therefore, since a SMM represents an isolated magnetic entity, it might be considered as the smallest practical unit for magnetic memories. The studying of a possibility of the switching of the spin states would incredibly increase the amount of information storable with respect to current devices. In order to have a deeper understanding of fundamental features and a major control on technological aspects, the time-dependent spin dynamics constitutes the key point. The micromagnetic model describes appropriately the dynamics of the magnetization(spin) in a magnetic nanostructures. In this model the magnetization(spin) is assumed to be a spatial- and time-dependent continuous function. The magnetization(spin) dynamics are described by the Landau-Lifshitz-Gilbert (LLG) equation including the energy contributions of the anisotropy, the exchange interaction, the magnetostatic interaction, and the Zeeman energy 14 . The microscopic result of the quantum modeling is the cluster spin models, which have been proved to be a powerful tool for approaching the extreme phenomenology of the ultrafast magnetization(spin) dynamics. Ab initio models for the general spin Hamiltonian can be efficient in providing insight on the local atomic scale values such as the local magnetic moment $\mu_{s}$, the local anisotropy $D$, or a pairwise exchange $J_{i j}$ in the magnetic molecule. The parameters of the theory, the exchange integrals and the anisotropy, are usually fitted to experiments or calculated from static density functional theory (DFT) ${ }^{15}$.

Switching between the LS and HS states can be done by varying the temperature, applying pressure or by light irradiation. In the first and the second case the population of the LS and HS states is fully determined by thermodynamic equilibrium and such transitions are called temperature- and pressure-induced spin crossover transitions ${ }^{16}$. In the case of light-induced spin crossover, after initial excitation to d-states or to metal-to-ligand charge transfer states, the system undergoes a complex cascade of intermediate transitions and eventually gets trapped in a non-equilibrium metastable state. The whole process is called light-induced excited spin state trapping (LIESST). The details of the LIESST process are still under debate $\underline{17}$.

We report here the possibility of a commutation of the CoOEP SMM between the two states of a spincrossover system by a pulsed magnetic field 18 . Choos- ing a cobalt-based spin-crossover system follows the idea that cobalt(II)[CoOEP] materials ${ }^{21}$ as well as iron(III) ones have metal-ligand bonds shorter than those in iron(II) materials, leading to faster relaxation dynamics between the HS and LS states. The molecule cobalt(II) $\left([\mathrm{Ar}] 3 d^{7} 4 s^{2}\right)$ may be either in the LS state $(\uparrow \downarrow \uparrow \downarrow \uparrow \downarrow \uparrow S=$ $1 / 2)$ or in the HS state $(\uparrow \downarrow \uparrow \downarrow \uparrow \uparrow \uparrow S=3 / 2)$, both of which are paramagnetic.

\section{THEORETICAL ASPECTS}

\section{A. The spin-dynamics simulations}

The dynamic behavior of a spin is determined by the equation of motion, which can be derived from the quantum theory with the general spin Hamiltonian $\widehat{H}_{\text {spin }}$ that calculated the spin structure of a magnetic molecule with Hamiltonian

$$
\widehat{H}_{s p i n}=\widehat{H}_{e x}+\widehat{H}_{a n}+\widehat{H}_{Z E E}+\widehat{H}_{a}(t) .
$$

The first term $\hat{H}_{e x}$ is the Heisenberg-Dirac Hamiltonian, which represents the isotropic exchange interaction, $H_{a n}$ is the exchange Hamiltonian the term due to the axial single-ion anisotropy, and $H_{Z E E}$ is the interaction between the spin system and the external magnetic field. In order to give a theoretical description of a magnetic molecule we exploit the irreducible tensor operator technique $\underline{20}$. For the study of the CoOEP magnetic properties, it is a good approximation to treat the electrons of one Co-atom as a single atomic spin which is interacting with its surroundings. Thus, one can be content with $\widehat{H}_{\text {spin }}$ without the first term $\hat{H}_{e x}$. We will consider an approximate treatment, restricting ourselves to the simplest, but very useful, approximation, known as mean field theory. This "mean field" Hamiltonian 21 , which describes the interaction of the spin $\widehat{\mathbf{S}}$ with the external magnetic field, given by its flux $\mathbf{H}_{e f f}$, can be expressed as:

$$
\widehat{H}_{\text {spin }}=-\gamma \mathbf{H}_{e f f} \widehat{\mathbf{S}} .
$$

The generally shaped formula is

$$
\mathbf{H}_{e f f}=-\frac{\partial \widehat{H}_{s p i n}}{\partial\langle\widehat{\mathbf{S}}\rangle} .
$$

Here the effective magnetic field $\mathbf{H}_{\text {eff }}$ is an external magnetic field $H_{z}$, the anisotropy fields $\mathbf{H}_{a n}$, the exchange interaction $\mathbf{H}_{e x}$ and external magnetic pulse field $\mathbf{H}_{\text {pulse }}(t)$. We use the "mean field" 21 approximation for $\mathbf{H}_{\text {eff }} \Rightarrow \mathbf{H}_{\text {eff }}^{\text {mean }}$ with replacement $\widehat{\mathbf{S}} \Rightarrow \mathbf{M}_{s}=\gamma\langle\widehat{\mathbf{S}}\rangle$. We call "mean" the mean field. (Historically it was often called the "molecular field"). It includes the average effect of the neighbors but neglects correlations between the spin and its neighbors. Since we have the one magnetic atom, this approach us quite suitable. Use (2) we 
obtain that ${ }^{22}$

$$
\frac{\partial\langle\widehat{\mathbf{S}}\rangle}{\partial t}=\frac{1}{1+\lambda^{2}}\langle\widehat{\mathbf{S}}\rangle \times \mathbf{H}_{e f f}^{\text {mean }}-\frac{\lambda}{1+\lambda^{2}}\langle\widehat{\mathbf{S}}\rangle \times\left(\langle\widehat{\mathbf{S}}\rangle \times \mathbf{H}_{e f f}^{\text {mean }}\right)
$$

The effective magnetic field $\mathbf{H}_{\text {eff }}^{\text {mean }}$ is given by the free magnetic energy variational with magnetization:

$$
\mathbf{H}_{e f f}^{\text {mean }}\left(\mathbf{M}_{s}, t\right)=-\frac{\delta F}{\delta \mathbf{M}_{s}}
$$

where $F$ is the free energy of the magnetic nanosystem

$$
F=-N k_{B} T \ln Z\left(H_{z}\right)
$$

with the partition function

$$
Z\left(H_{z}\right)=\sum_{M_{s}, \mu} \exp \left[-\epsilon_{\mu}\left(M_{s}\right) / k T\right] \sum_{M_{s}} \exp \left[-g_{e} M_{s} H_{z} / k T\right]
$$

- Here we have the energy levels $\epsilon_{\mu}\left(M_{s}\right)$ of the spinHamiltonian $\widehat{H}_{\text {spin }}$, which stay we diagonalizied $\widehat{H}_{\text {spin }}$.

A spin structure is defined only proceeding from the spin model of a molecule. Here we use to calculate a spin structure by the ITO method within the generalized spin Hamiltonian $\widehat{H}_{\text {spin }}$.

Once we have the energy levels of the spinHamiltonian, we can evaluate a different thermodynamic properties of the system as the magnetization, the magnetic susceptibility, and the magnetic specific heat. Since in further researches the anisotropic part of a molecule will be only scalar, the magnetic properties of the anisotropic system do not depend on the direction of the magnetic field. Thus we can consider the external magnetic field $H_{z}$ directed along arbitrary axis $z$ of the molecule coordinate frame that is chosen as a spin quantization axis. In this case the energies of the system will be $\epsilon_{\mu}\left(M_{s}\right)+g_{e} M_{s} H_{z}$, where $\epsilon_{\mu}\left(M_{s}\right)$ are the eigenvalues of the spin-Hamiltonian containing the magnetic exchange and the double exchange contributions (index $\mu$ runs over the energy levels with given total spin protection $\mathbf{M}_{s}$ ). Using this expression one can evaluate the magnetization $\mathbf{M}_{s}$ by the standart thermodinamic definitions:

$$
\mathbf{M}_{s}=\frac{\partial F\left(M_{s}, H_{z}\right)}{\partial \mathbf{H}}=N k T \frac{\partial \ln Z}{\partial \mathbf{H}}
$$

The effective field $\mathbf{H}_{\text {eff }}^{\text {mean }}$ can be derived from the free energy functional

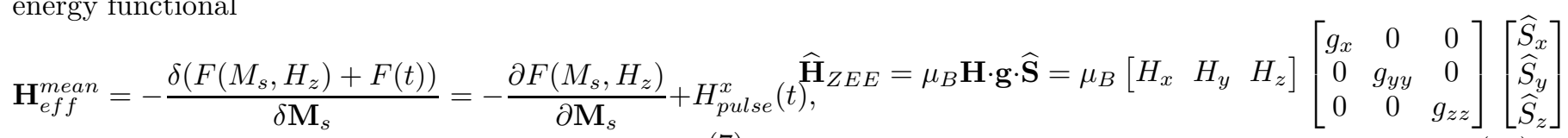

We have derived a general form of the time-dependend spin equation for a system of the spins precessing in an effective magnetic field with specifying the interactions in the magnetic molecule.

\section{B. Calculations of spin-Hamiltonian parameters}

The present research is devoted to study a time-dependent behavior of the Co-octaethylporphyrin molecule after applying an external magnetic field in a picoseconds time span. In order to optimize the molecule geometry and determinate the spin-Hamiltonian parameters - zero-field splitting (ZFS, D-tensor), g-tensor, the exchange parameters $J_{i j}$, and the single-ion anisotropy parameters, we performed Density Functional Theory (DFT) calculations with taking into account the spinorbit coupling effects using ADF package ${ }^{23,24}$. The calculations were performed with the Perdew-Burke-Ernzerhof (PBE) exchange-correlation functional and the ZORA scalar relativistic Hamiltonian, using an all-electron valence triple zeta with a one polarization function (TZP) basis set and a 'Good Becke' grid. The zero-field splitting (ZFS) of the Co OEP ground state was found to be equal $1.32 \mathrm{meV}$. ZFS is the breaking of degeneracy of the ground state that is not described by a standard non-relativistic Hamiltonian. As calculated by ADF, the ZFS is that exhibited by molecules whose ground state characterized by the spin $\mathrm{S}>1 / 2$ and absence of a spatial degeneracy. This type of ZFS has two contributions, second-order spin-orbit coupling and spin-spin coupling. In the present implementation only the spin-orbit coupling term is included. In our study the ZFS was calculated in combination with GGA PBE functional. In order to calculate ZFS the relativistic scalar Zero Order Regular Approximation (ZORA) option ${ }^{25}$ was included. The ZFS Hamiltonian is given by

$$
\widehat{H}_{Z F S}=\widehat{\mathbf{S}} \cdot \mathbf{D} \cdot \widehat{\mathbf{S}}
$$

where $\widehat{\mathbf{S}}$ is the vector operator for the effective spin of Co atom, and D is the second-order anisotropy tensor. After diagonalization in the magnetic axis frames, the Hamiltonian becomes

$$
\widehat{H}_{Z F S}=D \widehat{S}_{z}^{2}+E\left(\widehat{S}_{x}^{2}-\widehat{S}_{y}^{2}\right)
$$

The parameter D is called the axial ZFS parameter and $\mathrm{E}$ is the rhombic ZFS parameter, which vanishes for high symmetry molecules. Parameter D has to be negative for the magnetic bistability assosiated with SMMs. When a molecule is placed in a magnetic field $\mathbf{H}$, the electron's energies will depend on its magnetic moment value $\mathbf{M}_{s}$. To account for the anisotropy of the Zeeman response to an applied magnetic field, an "effective" Zeeman Hamiltonian using a so-called "g tensor" is used

The g-tensor was calculated in a spin-orbit coupled spin unrestricted relativistic ZORA approach. The degenerate perturbation theory was used with the external magnetic field as perturbation. 


\section{RESULTS AND DISCUSSION}

The most important aspect to the establishment of real devices based on SMMs, e.g. high density rewritable memories, is to achieve such conditions under that externally driven system could be switched between two stable LS and HS spin states and would remain in each of these states for a certain period of time. The LS/HS bi-stability is connected with small energy $\left(\Delta E_{H S-L S}=2.133 \mathrm{meV}\right)$ magnitudes involved in the switching between these two spin states (Figure 1a). Much research is currently focused on the study of photoswitching mechanism between LS and HS states, changes in the geometric and electronic structure of molecules in these states, the magnetic properties of SMMs. However, we found no studies on the time-dependent spin dynamics of spin-crossover systems. In this paper we focus on the behavior of the spin of the molecules under the influence of external stimuli and finding the conditions under which the system would be able to stay in one of the bistable states arbitrarily long time. Since the photo-induced states of SMMs are characterized by a short lifetime, we effect on the system being studied by a short magnetic pulse of high amplitude. In the experimental study $\stackrel{18}{\frac{18}{}}$, it was shown that the application of an external magnetic field of $32 T$ to spin-crossover solid system $\mathrm{Co}\left(\mathrm{H}_{2}(\mathrm{fsa})_{2} \mathrm{en}\right)(\mathrm{py})_{2}$ takes place an irreversible and quasicomplete transition from the HS to the LS state. This also means that after applying a perturbation the system can remain in HS state for a long time. In this paper, we have developed an approach for the study of the spin dynamics of spin-crossover systems and apply it to one of the intensively studied objects from a number of SMMs the CoOEP molecule. The chemical structure of CoOEP molecule is exhibited in Fig. 1(c). Fig 2 shows the magnetic susceptibility plotted as the product $\chi T$ versus temperature for the CoOEP molecule. The temperature variation of the magnetic susceptibility $\chi T$ showing the abrupt of the thermal spin transition with the jump between two spin states occurs at temperature of $T_{1 / 2}=40 \mathrm{~K}$.

By irradiating LS molecules with visible light, HS molecule can be generated because of the efficient electronic decay of excited state via Inter System Crossing towards matastable HS state. This effect is now referred as LIESST (Light Induced Electronic Spin State Trapping) in the literature ${ }^{26}$. Once irradiation is stopped, the system relaxes to the thermal equilibrium state LS. One important LIESST-result is the decoupling of different degrees of freedom during the electronic spin state trapping and the transfer of excess energy to molecular vibration modes. Contrary to the LIESST, here considered in some detail the quantum dynamics of the HS spin state trapping and impacts on it of a short magnetic pulse.

We present here a theoretical approach to the organic magnetic CoOEP-molecule in terms of numerically solvable the time-dependent LLG-model with a general

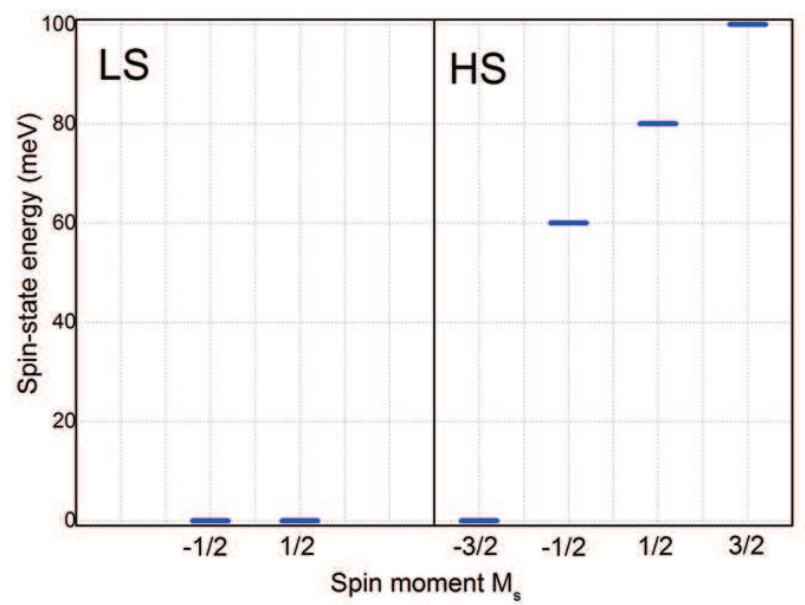

(a)

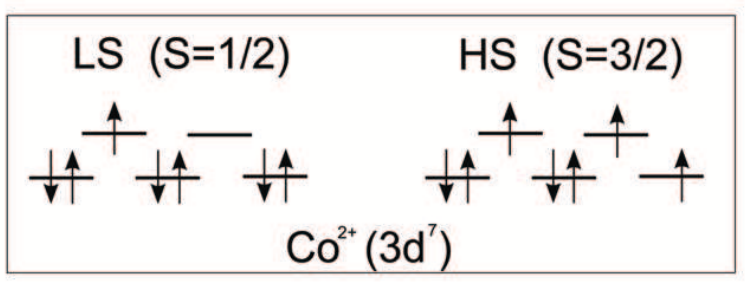

(b)

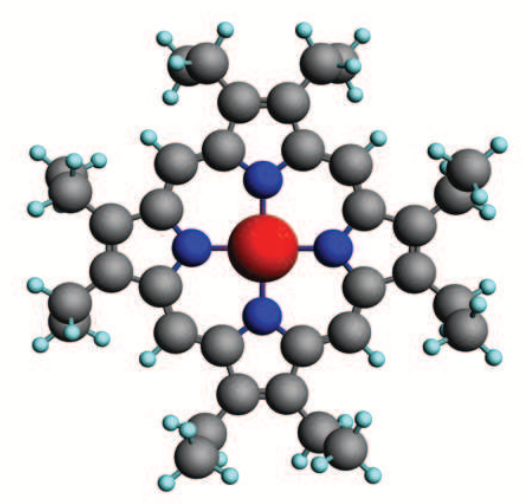

(c)

FIG. 1: (Color online) (a)Spin structure of CoOEP molecule in a spin crossover system HS-LS states;(b)HS and LS states with $3 d^{7}$ configuration of $C o^{I I}$ in a tetrahedral field;(c)The molecular structure of CoOEP.

spin Hamiltonian in the context of the ITO model. In practice, these models apply to the quantum spin-state switching in the Co-octaethylporphyrin molecular magnet with a picosecond pulsed magnetic field and provide an understanding of a spin-crossover phenomenon in these molecule. In the calculations by LLG-method was used the next parameters: $T_{\text {pulse }}=0.17 p s$ (ultrashort $\mathrm{THz}$ pulse); time of start pulse is $37.4 \mathrm{ps}$; the height of 


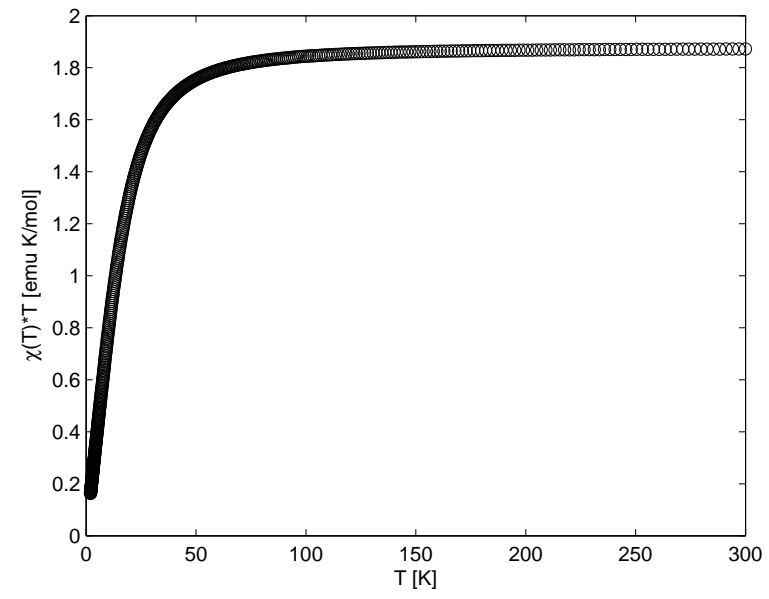

FIG. 2: (Color online) The magnetic susceptibility product $\chi$ $\mathrm{T}$.
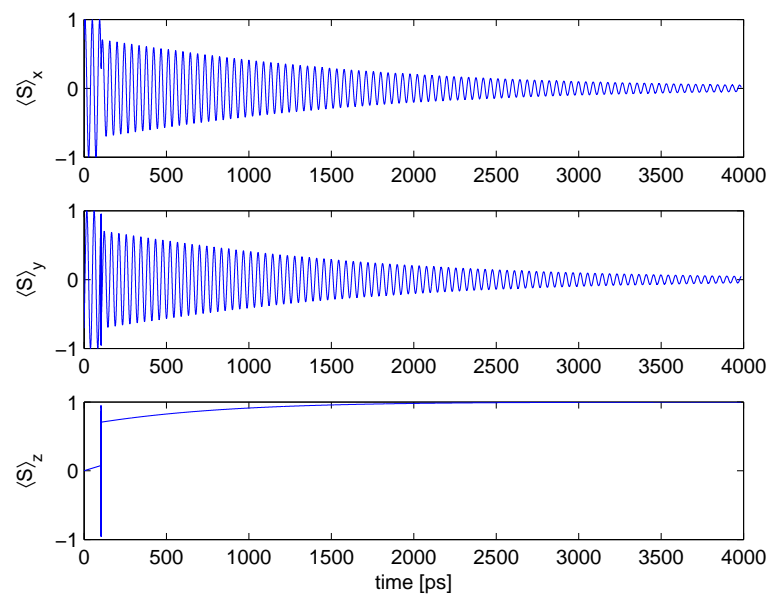

FIG. 3: (Color online) The spin dynamics for the CoOEP molecule with magnetic pulse $(S=1 / 2)$.

pulse is $36.8 T$. We solved the LLG equation for two different states $\mathrm{S}=1 / 2, t_{2 g}^{6} e_{g}^{1}(\mathrm{LS})$ and $\mathrm{S}=3 / 2, t_{2 g}^{5} e_{g}^{2}$ (HS). Under the influence of the constant Zeeman magnetic field $0.1 T$, after a certain period of time, which is determined in case of a single molecule by the size of ZFS, the time-dependent spin structure is formed. In the time of "saturation" of the system we apply the external stimuli of a magnetic pulse. The system in the LS state behaves classically without the application of the pulse. Under the influence of the $36.8 T$ pulse a spin switching on the pulse duration of $100 \mathrm{fs}$ is happened, after which the system returns to the ground state (Fig 3). In the case of the HS state of the system the situation is changing drastically. Under the influence of the pulse a spin switching occurs as well, but the system remains in this state
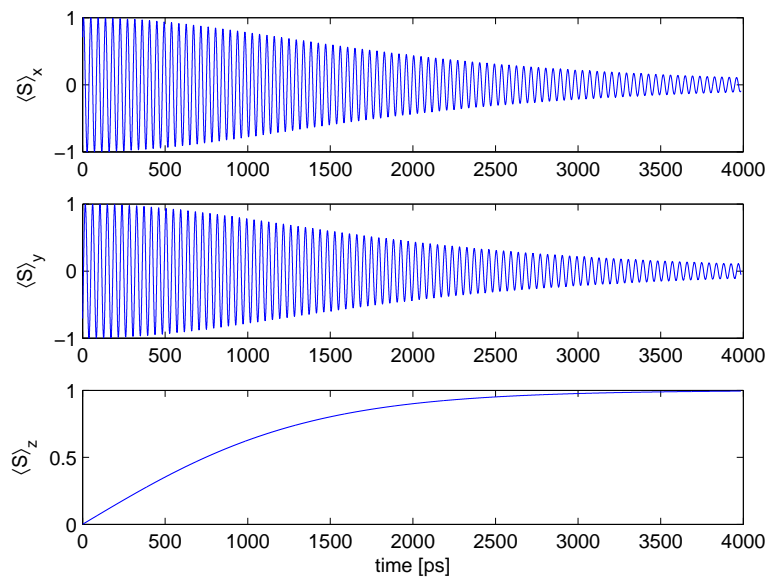

FIG. 4: (Color online) The spin dynamics for the CoOEP molecule without magnetic pulse $(\mathrm{S}=1 / 2)$

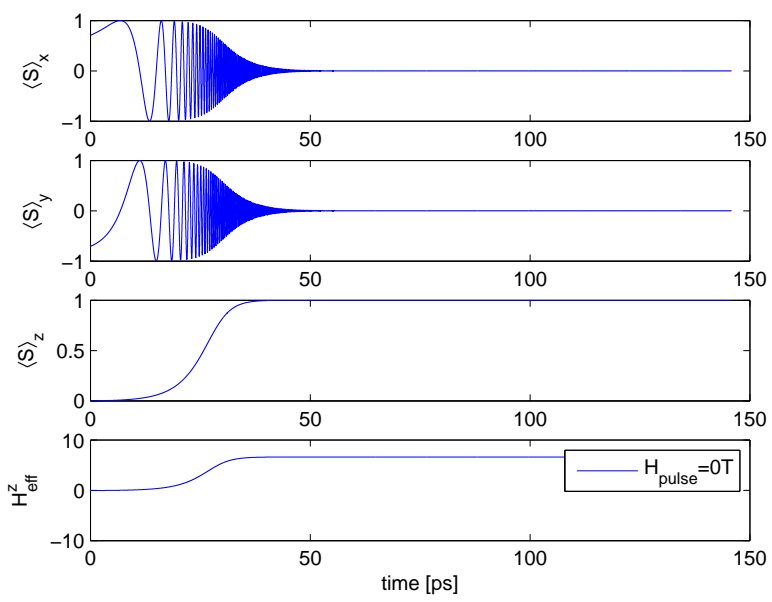

FIG. 5: (Color online) The spin dynamics for CoOEP molecule without magnetic pulse $(S=3 / 2)$.

for a long time (Fig [6). With increasing magnetic field up to $36.8 T$ a spin switch occurs and the $H_{\text {eff }}$ sign is changed. In our approach $H_{e f f}$ is the $\langle\widehat{\mathbf{S}}\rangle$ derivative of the spin-Hamiltonian. In our case, the Hamiltonian (11) has no $H_{e x}$ term, because there is only one transition metal atom in CoOEP molecule and, accordingly, the spin-spin interaction is absent. Thus, the anisotropic $H_{a n}$ term is only a nonconstant one, which is quadratic in $\langle\widehat{\mathbf{S}}\rangle$ (9) and, therefore, $H_{\text {eff }}$ is proportional to $H_{\text {eff }}$ is the $\langle\widehat{\mathbf{S}}\rangle$. Qualitatively, the $H_{\text {eff }}$ behavior is the same as the $\langle\widehat{\mathbf{S}}\rangle$, which is seeing from the Fig 5 and 6 , 


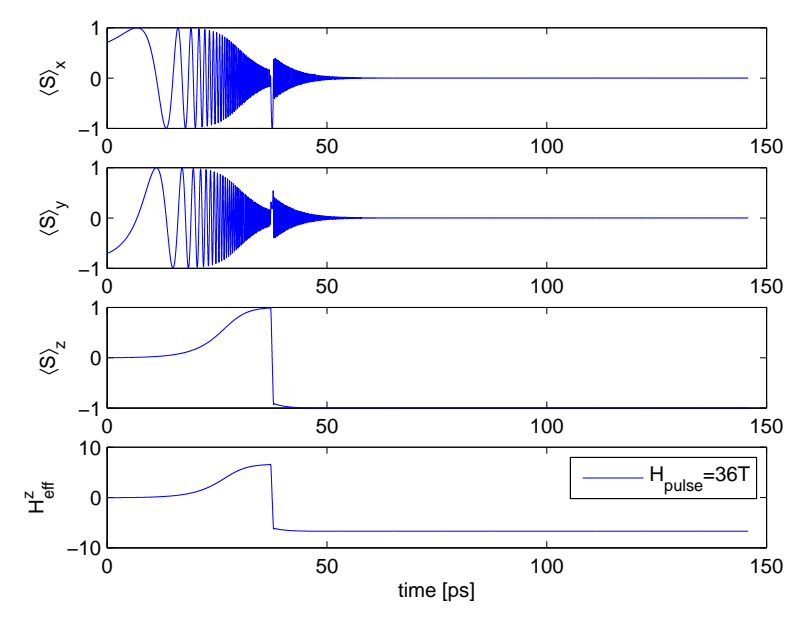

FIG. 6: (Color online) The spin dynamics for the CoOEP molecule with magnetic pulse $(S=3 / 2)$.

\section{CONCLUDING REMARKS}

In the paper we present the results of the theoretical study of the spin behavior in the CoOEP molecule under influence of high magnetic pulse. Based on the LLG equation we described the spin dynamics of the molecule in the LS and HS states. We found that the spin-crossover CoOEP molecule undergo the spin switching between LS and HS states after applying the external stimuli of a short magnetic pulse of $36.8 \mathrm{~T}$. The temperature dependency of magnetic susceptibility shows an abrupt thermal spin transition between two spin states at $40 \mathrm{~K}$. We also found the difference in the spin dynamics for two spin states of CoOEP molecule. Thus, after the spin switching under the magnetic pulse the system in the LS state reverts to its ground state, whereas the system in the HS state after the spin switching remains in the excited state for a long time. This behavior gives us the possibility to assign such system as an appropriate candidate for the SMM and opens a way to realize a controlled spin switching in real devices for spintronics.

\section{Acknowledgments}

The part of this research has been supported by Russian Ministry of Science, Grant No. 213.01-11/2014-6 (for V.L.M.) and RFMEFI 58714X0002 (for O.V.F.). The work is partially supported by the Max-Planck Gesellschaft during the stay of O.V.F. in MPI (Halle) where this work was completed. The authors are indebted to Prof. Alexander Soldatov and Dr. Alexander Guda for detailed discussion.
1 F. Troiania and M. Affronte, Chem.Soc.Rev. 40, 3119 (2011).

2 A. P. de Silva and S. Uchiyama, Nat. Nanotechnol, 2, 399 (2007).

3 W. Hübner, S. Kersten, and G. Lefkidis, Phys. Rev.B 79, 184431 (2009).

4 D. A. Allwood, G. Xiong, M. D. Cooke, C. C. Faulkner, D. Atkinson, N. Vernier, and R. P. Cowburn, Science, 296, 2003 (2002).

${ }^{5}$ B. Ferguson and X.-C. Zhang, Nat. Mater. 1, 26 (2002).

${ }^{6}$ M. Tonouchi, Nat. Photonics, 1, 97 (2007).

7 C. Vicario, C. Ruchert, F. Ardana-Lamas, P. M. Derlet, B. Tudu, J. Luning, and C. P. Hauri, Nat. Photonics, 7, 720 (2013).

8 C. Vicario, B. Monoszlai, and C. P. Hauri, Phys. Rev. Lett. 112, 213901 (2014).

9 R. Wieser, E. Y. Vedmedenko and R. Wiesendanger, Phys.Rev.Lett. 106, 067204 (2011).

10 O.V.Farberovich, V.L.Mazalova, arXiv:1410.1658v1 [cond-mat.mes-hall] 7 Oct.2014.

11 A. Tsiatmas, E. Atmatzakis, N. Papasimakis, V. Fedotov, B. Luk'yanchuk, N.I. Zheludev and F. Javier Garcia de Abajo, arXiv:1303.6072 v 1 cond-mat.mes-hall]25 Mar.2013.

12 A. Kirilyuk, A.V. Kimel and T. Rasing, Rev. Mod. Phys. 82, 2731 (2010).

13 W. Linert and M. Verdaguer, eds., Molecular Magnets (Springer-Verlag, Wien, 2003),

14 B. Guo and S. Ding, Landau-Lifshitz equations (World Sci- entific, Singapore, 2008).

15 V.V.Maslyuk, I. Mertig, O.V. Farberovich, A. Tarantul and B. Tsukerblat, Eur.J.Inorg.Chem. 2013, 1897 (2013).

16 Spin-Crossover Materials, edited by M. Halcrow (Wiley, West Sussex, 2013).

17 M. Cammarata, R. Bertoni, M. Lorenc, H. Cailleau, S. Di Matteo, C. Mauriac, S.F. Matar, H. Lemke, M. Chollet, S. Ravy, C. Laulh, J.-F. Ltard and E. Collet, Phys. Rev. Lett. 113, 227402 (2014).

18 A. Bousseksou, K. Boukheddaden, M. Goiran, C. Consejo, M-L. Boillot and J-P. Tuchagues, Phys. Rev.B 65, 172412 (2002).

19 M. Bernien, X-ray absorption spectroscopy of Fe complexes on surfaces. PhD Thesis, Freie Universitt Berlin (2010).

20 J.J. Borras-Almenar, J.M. Clemente-Juan, E. Coronado and B.S. Tsukerblat, Inorg.Chem.38, 6081 (1999).

21 D.A. Garanin, Phys. Rev.B 55, 3050 (1997).

22 R. Wieser, arXiv:1302.1985 1 [cond-mat.mes-hall] 8 Feb 2013.

23 G. te Velde, F. M. Bickelhaupt, S.J.A. van Gisbergen, C. Fonseca Guerra, E.J. Baerends, J.G. Snijders and T. Ziegler, J. Comput. Chem. 22, 931 (2001).

24 ADF2014, SCM, Theoretical Chemistry, Vrije Universiteit, Amsterdam, The Netherlands, http://www.scm.com

25 E. van Lenthe, A. E. Ehlers and E. J. Baerends J. Chem. Phys. 110, 8943 (1999).

26 S.F. Matar, Ph. Guionneau and G. Chastanet, Int. J. Mol. Sci., 16, 4007 (2015). 